\title{
Isolation and Detection of Alpha Linolenic Acid from Silkworm Pupal Residue Oil (Bombyx mori L.) Using HPLC
}

\author{
B.S. Vidhathri ${ }^{1}$, V.R. Ramakrishna Parama ${ }^{2}$, C.T. Subbarayappa ${ }^{2}$, \\ T.K. Narayanaswamy ${ }^{1}$, R. Muthuraju ${ }^{3}$, D.S. Mahesh ${ }^{1 *}$ and D.N. Vidyashree ${ }^{3}$ \\ ${ }^{1}$ Department of Sericulture, UAS, GKVK, Karnataka, India \\ ${ }^{2}$ Department of Soil Science and Agricultural Chemistry, UAS, GKVK, Karnataka, India \\ ${ }^{3}$ Department of Agricultural Microbiology, UAS, GKVK, Karnataka, India \\ *Corresponding author
}

\section{A B S T R A C T}

The omega fatty acids are a group of compounds that are included under essential fatty acids. Alpha-linolenic acid is an essential omega-3 fatty acid, needed for normal human

Keywords

Alpha-linolenic acid, Silkworm pupal residue, Extraction, Isolation.

Article Info

Accepted:

21 June 2017

Available Online:

10 July 2017 growth and development. The silkworm pupae oil contains $25.7 \%$ of alpha linolenic acid. There are many methods adopted for purification and quantification of ALA. However, HPLC technique was adopted as an established method of quantifying ALA from vegetable oils. This method has been modified to quantify ALA from silkworm pupal residue oil. Alpha linolenic acid was isolated by using two step crystallization methods. Silkworm pupal oil contains both unsaturated and polyunsaturated fatty acids. Unsaturated fatty acids are removed in first step of crystallization at $4^{\circ} \mathrm{C}$ for about 24 hours. Polyunsaturated fatty acids were crystallized at $-18^{\circ} \mathrm{C}$. Isolated alpha linolenic acid was analyzed by HPLC against sigma Aldrich standard. On the basis of UV/VIS spectra of fatty acids, the poly unsaturated fatty acids have absorption peaks about $210 \mathrm{~nm}$ to $300 \mathrm{~nm}$. The polyunsaturated fatty acids (ALA) absorption peaks were observed at $210 \mathrm{~nm}$. Monitoring of ALA was carried out at $210 \mathrm{~nm}$. The ALA in the sample was identified by comparing the retention time with that of respective standard. The retention time of ALA and sample was 4.39. Quantification was carried out by integration of the peaks using external standard.

\section{Introduction}

Alpha-linolenic acid (ALA), which belongs to a group of fatty acids named omega-3 fatty acids, is an essential fatty acid. Intake of omega-3 fatty acids reduces risk of fatal coronary heart events, perhaps by stabilizing myocardium and reducing risk of fatal arrhythmias. The wide variety of ALAexerted biological actions may have relevance to their involvements in several physiological and pathological processes. ALA defect is closely related to the prevalence of hypertension, diabetes mellitus, coronary heart disease, schizophrenia, Alzheimer's disease, athero-sclerosis and cancer, thus depicting the importance of adequate intake of ALA in daily life. The silkworm spins a silk cocoon as protection for pupa. Duration of pupal stage is 12-15 days. In 2014, world production of reel able silk cocoons was about $4,85,000$ MT. By subtracting the amount of raw silk $(1,61,000 \mathrm{MT})$, it can be assumed that $3,24,000 \mathrm{MT}$ of fresh pupae $(65,000 \mathrm{MT}$ 
dry) was produced (Anonymous, 2014). India and Karnataka produces, 1, 39,871 MT and 55,957 MT of green mulberry cocoons out of which 20 per cent is dry pupae weight (Anonymous, 2013).

The fatty acid composition of silkworm pupae, Bombyx mori L was reported (Nakasone and Ito, 1967; Zhou and Han, 2006). Variations in the level of ALA (traces to $40 \%$ of total fatty acids) reported (Shivshanker et al., 2006).

In view of the pharmaceutical application of ALA, the preparation of ALA from desilked silkworm pupae is thus of essential importance.

\section{Materials and Methods}

\section{Silkworm pupal residue}

Silkworm pupal residue samples were collected from reeling units of Channapatna and Ramanagara of Ramanagara district, Vijayapura of Bengaluru rural district and shidlaghatta of Chikkaballapura district of Karnataka state. They were categorised into five catagories viz., immature, diseased, healthy, broken and mixed silkworm pupal residue sample. From these samples the healthy pupal residue catagories was used for isolation of Alpha linlenic acid.

\section{Chemicals and reagents}

Standard alpha linolenic acid was procured from Sigma Chemical Co. (St. Louis, MO, USA). All reagents used were of analytical grade, except methanol and acetonitrile, which were of HPLC grade. Standard ALA was accurately measured and prepared by dissolving it in methanol. It was then diluted to appropriate concentration ranges for the establishment of calibration curve. All solutions were stored at $4^{\circ} \mathrm{C}$.

\section{Preparation of ALA}

\section{Extraction of pupal oil}

The healthy pupal residue samples were dried at 55 to $60^{\circ} \mathrm{C}$ till constant weight. The dried pupal residue was powdered.

Silkworm pupal oil was extracted by using soxhlet apparatus taking $40 \mathrm{~g}$ of dried pupal powder in $270 \mathrm{ml}$ of solvents mixture (petroleum ether and methanol) the sample was distilled for about 5 hrs.

\section{Isolation of alpha-linolenic acid from pupal oil}

The pupal oil was then saponified in $50 \mathrm{ml}$ of methanol containing $0.3 \mathrm{M} \mathrm{KOH} 90^{\circ} \mathrm{C}$ under a gentle stream of $\mathrm{N}_{2}$ for $2 \mathrm{~h}$. After the removal of methanol in a rotary evaporator, the mixture was acidified to $\mathrm{pH} 1.0$ using $1 \mathrm{M}$ $\mathrm{H}_{2} \mathrm{SO}_{4}$. The top layer containing free fatty acids was washed three times with the same volume of distilled water. Isolation of alpha linolenic acid was carried out using two-step crystallization. The free fatty acid mixture was dissolved into three volumes of methanol. The crystallization was carried out at $48{ }^{\circ} \mathrm{C}$ to remove the saturated fatty acids and then at $18^{\circ} \mathrm{C}$ to obtain alpha linolenic acid. The process was repeated twice (Yang et al., 2005).

\section{Chemical analysis}

The content of ALA was analyzed by highperformance liquid chromatography (HPLC) using a C18 column $(4.6 \times 250 \mathrm{~mm} ; 5 \mu \mathrm{M})$. Solvents acetonitrile: methenol: n-Hexane in the ratio $90: 8: 2$.flowrate $1 \mathrm{ml} / \mathrm{min}$ at $210 \mathrm{~nm}$. Runtime 10 min, only B pump was used.

Five standards viz., 2, 4, 6, 8 and 10 micro liters we prepared with methanol for standardization of Alpha linolenic acid. The 
quantification of ALA in silkworm pupal residue oil was determined by back calculation method. Similar procedure was reported for Quantification of fatty acids from vegetable oils (Guarrasi et al., 2010).

\section{Results and Discussion}

On the basis of UV/VIS spectra of fatty acids poly unsaturated fatty acids have absorption peaks at about $210 \mathrm{~nm}$ to $300 \mathrm{~nm}$.The polyunsaturated fatty acids (ALA) absorption peaks were observed at $210 \mathrm{~nm}$. Hence, monitoring of ALA was carried out at 210 $\mathrm{nm}$. The HPLC chromatogram of standard ALA and sample was shown (Fig. 1). Further, the HPLC chromatogram peaks comparison for five standards with different dilutions along with sample was shown in figure 2. The peak of sample ALA from pupal oil was symmetrical. The ALA in the sample was identified by comparing with retention time with that of respective standard. The retention time of ALA and sample was 4.39; the identification was carried out by integration of peaks using external standards (Figs. 1 and 2). Peruse of available literature revealed that the similar method was not tried for isolation detection of ALA from silkworm pupal residue oil. Hence, due to paucity of literature the same has not been discussed. However Wang et al., (2010) reported that urea clathration method was used to isolate and analyze alpha linolenic acid from silkworm pupal oil.

Fig.1 HPLC-UV Chromatogram of ALA standard and sample from silkworm pupal oil
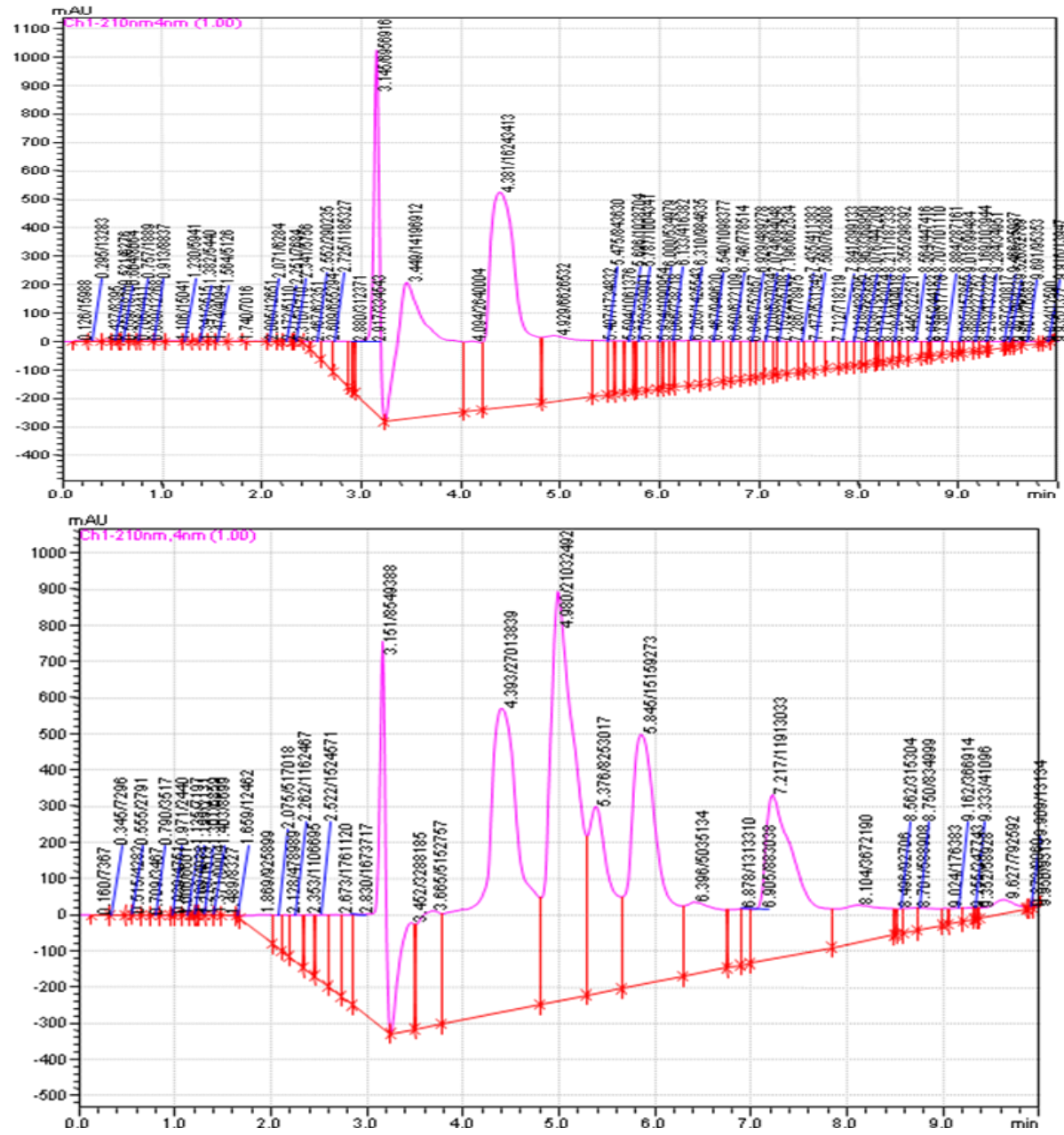
Fig.2 Comparison of sample of alpha linolenic acid with different dilution of standard ALA

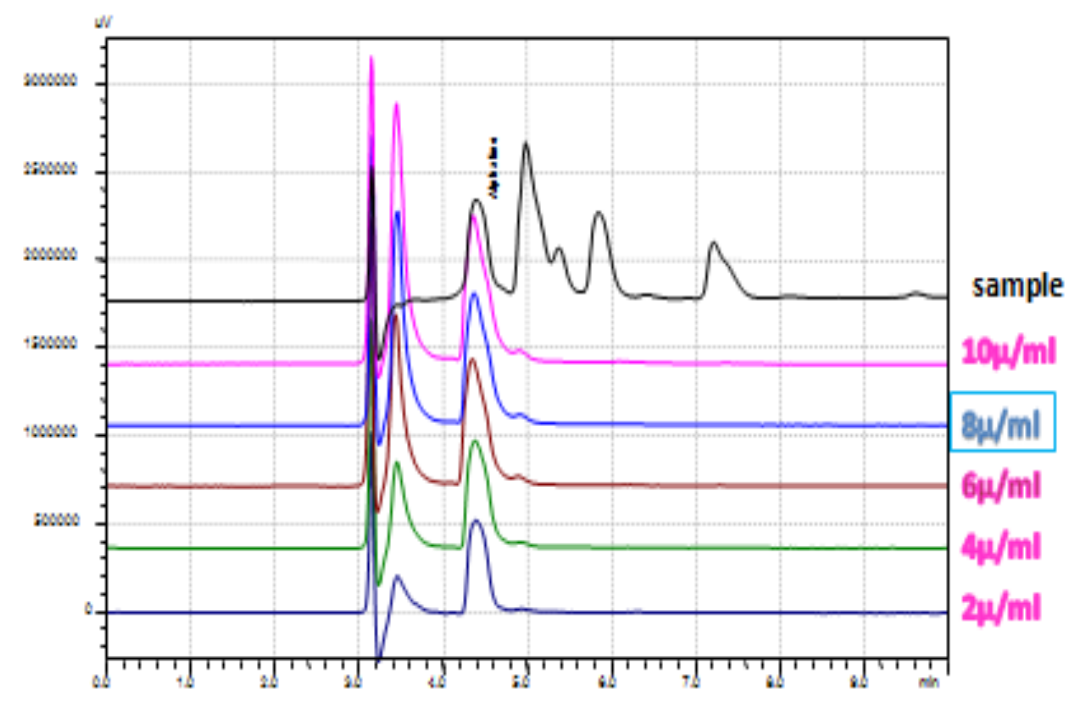

Fig.3 Calibration data for ALA

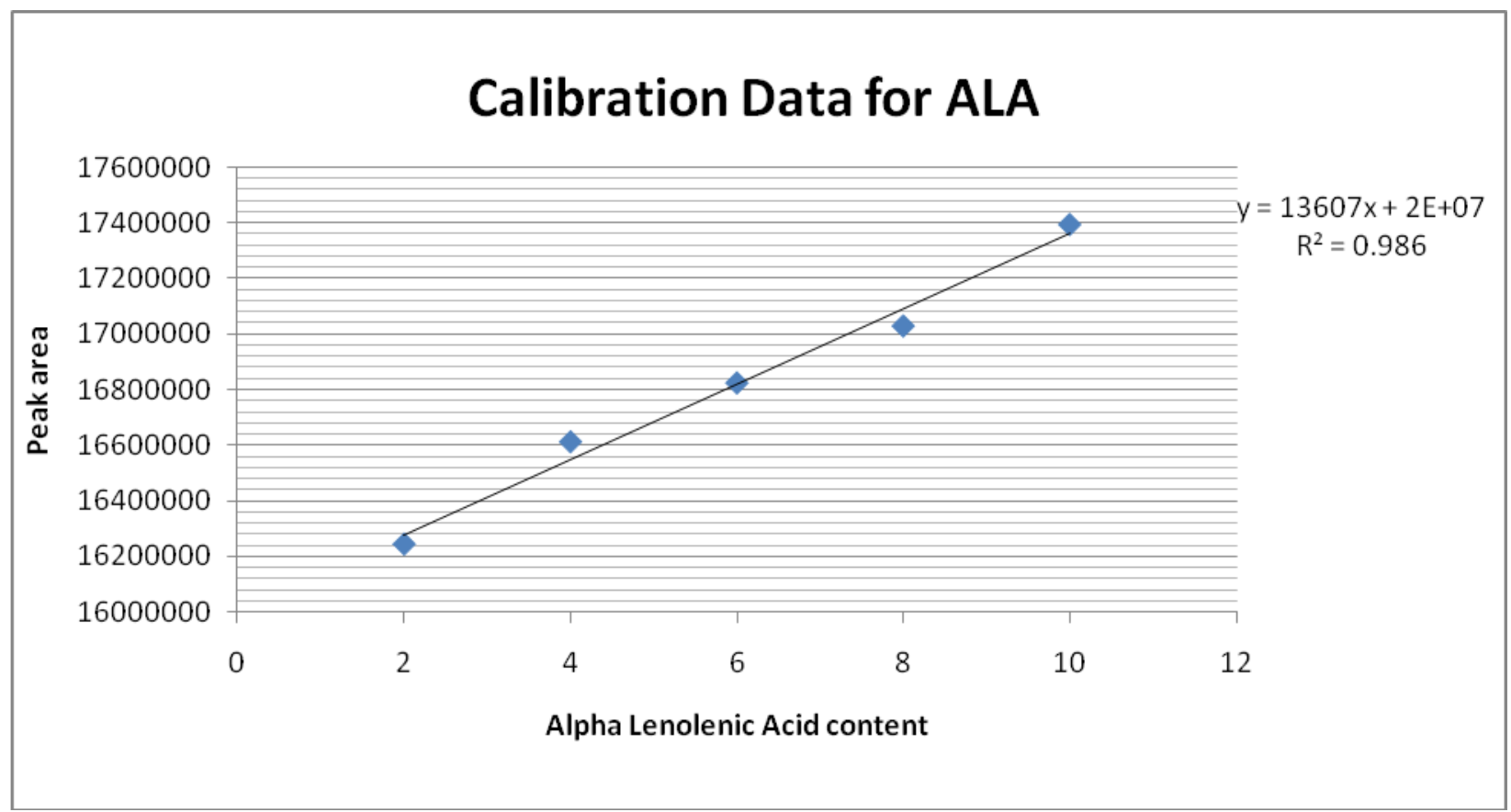

\section{Calibration data}

Alpha linolenic acid in the samples analyzed was identified by comparing its retention time with its standard. Quantification was carried out by integration of the peaks using external standard (Fig. 3). The results of calibration data for ALA are presented in figure 3.
Response (peak area, Y) was then plotted against concentration (X, micro liter) and five point calibration curves were found to be linear which indicated that the developed analytical method is precise and sensitive for determining the content of ALA in test sample. 
The modified HPLC method in the present study yielded $6 \%$ of ALA from silkworm pupal residue oil. On the contrary Guarrasi et al., (2010) reported that 0.8 to $1.3 \%$ ALA was obtained from vegetable oils through HPLC method.

\section{Acknowledgement}

The authors greatly acknowledge the financial assistance provided through DBT funded project entitled "characterization of silkworm pupal bioprotein and processing it for value addition" from DBT, New Delhi.

\section{References}

Anonymous, 2013, Sericulture and silk industry statistics - 2012, Central Silk Board, Bengaluru: 20-21

Anonymous, 2014, FAO Statistical Yearbook, Food and Agriculture Organization of the United Nations Regional Office for Europe and Central Asia, Budapest: $110-112$

Guarrasi, M. R., Mangione, Sanfraello, V., Martorana, V. and Bulone, D., 2010, Quantification of underivatized fatty acids from vegetable oils by HPLC with UV detection. $J$ chromatographic scie
48: 663-668.

Nakasone, S. and Ito, T., 1967, Fatty acid composition of the silkworm, Bombyx mori L. J. Insect. Physiol. 13: 12371246.

Shiva Shankar, K., Shireesha, K., Kanjilal, S., Sambharaju V. L., KUMAR N., Srinivas, C., Jammy V. K. R. and Prasad R. B. N. 2006. Isolation and characterization of neutral lipids of desilked eri silkworm pupae grown on castor and tapioca leaves. J. Agric. Food Chem., 54 (9): 3305 -3309.

Wang, J., Fu- A Wu., Yao Liang and Mang, M., 2010, Process of optimization for the enrichment of alpha linolenic acid from silkworm pupal oil using response surface methodology. African $J$ biotechnology, 9(20): 2956-2964.

Yang, L., Leung, Y. K., Cao, Y., Huang, Y., Ratnayke, W. M. N. and Chen Zhen Yu, 2005, Alpha linolenic acid but not conjugated linolenic acid is hypercholesterolaemic in hamsters. British j Nut, 93:433-438.

Zhou and Han, 2006, Proximate amino acid and mineral composition of pupae of the silkworm, Antheraea pernyi in China. $J$. Food Compost Anal., 19: 850-853.

\section{How to cite this article:}

Vidhathri, B.S., V.R. Ramakrishna Parama, C.T. Subbarayappa, T.K. Narayanaswamy, R. Muthuraju, D.S. Mahesh and Vidyashree, D.N. 2017. Isolation and Detection of Alpha Linolenic Acid from Silkworm Pupal Residue Oil (Bombyx mori L.) using HPLC. Int.J.Curr.Microbiol.App.Sci. 6(7): 2202-2206. doi: https://doi.org/10.20546/ijcmas.2017.607.258 